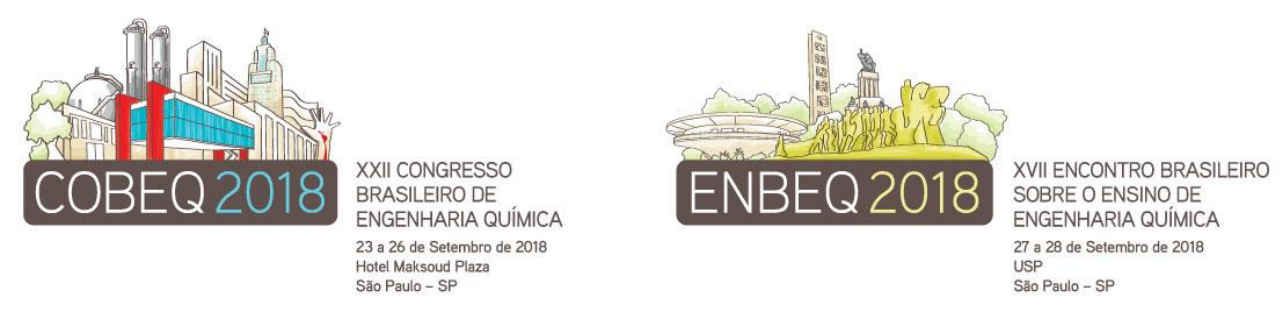

\title{
MODELAGEM DE CRISTALIZAÇÃO DE PARAFINA COMO MATERIAL DE MUDANÇA DE FASE APLICADA A ADSORÇÃO DE METANO.
}

\author{
COSTA A. B. ${ }^{1}$, DE MARTINO, V. R. DE MARTINO ${ }^{1}$, V. R., PANTOJA ${ }^{1}$, C. E., DE \\ PAIVA, J. L. ${ }^{1}$, SECKLER, M. M. ${ }^{1}$. \\ ${ }^{1}$ Universidade de São Paulo, Departamento de Engenharia Química \\ E-mail para contato: adriano.bonangelo@usp.br
}

\begin{abstract}
RESUMO - A adsorção é uma tecnologia que permite armazenar gás natural sob pressões relativamente baixas. Ao acrescentar ao leito materiais de mudança de fase, do inglês phase change materials (PCM), o aumento da temperatura associado à adsorção é minimizado, o que permite aumentar a capacidade de estocagem de gás. A taxa de transporte de calor através do PCM durante a cristalização não é bem conhecida, pois depende da morfologia da camada em formação. A fim de antecipar estudos experimentais, simulou-se a troca de calor de PCM parafínico em aparato de dedo frio. O tempo para a formação de um depósito de $15 \mathrm{~mm}$ de espessura de parafina, com condutividade de 0,240 W/m.K, foi de 33 min e com a metade da condutividade foi de $48 \mathrm{~min}$. Caso a forma cristalina modifique-se, a mudança na condutividade influenciará no tempo de cristalização do PCM e consequentemente na velocidade de absorção ou liberação de energia durante a mudança de fase.
\end{abstract}

\section{INTRODUÇÃO}

O gás natural como combustível tem atraído grande atenção devido à instabilidade do mercado de petróleo e ao aumento das rigorosas regulamentações ambientais. O gás natural adsorvido, do inglês adsorbed natural gas (ANG), é uma tecnologia na qual o gás é armazenado na superfície de material poroso a pressões relativamente baixas. Além disso, ele permite o design flexível do tanque de armazenamento; tem um baixo custo e proporciona operação segura, especialmente quando é usado nas aplicações de transporte. Porém o processo de adsorção é exotérmico, aumentando a temperatura do meio adsorvente e reduzindo a eficiência na estocagem de gás (EL-SHARKAWY et al., 2015).

O trabalho de Li e Li (2015) propôs aplicação de materiais de mudança de fase, do inglês phase change materials (PCM), para a estocagem de gás natural, visando reduzir o problema que o aumento da temperatura causa devido à adsorção, os PCMs estocam energia em suas ligações químicas e a energia é liberada durante a cristalização e absorvida durante sua fusão. Dependendo da vazão de alimentação e do adsorvente utilizado, segundo Zakaria e George (2011), as temperaturas, a partir de $25^{\circ} \mathrm{C}$, podem aumentar até aproximadamente 75 ${ }^{\circ} \mathrm{C}$. 


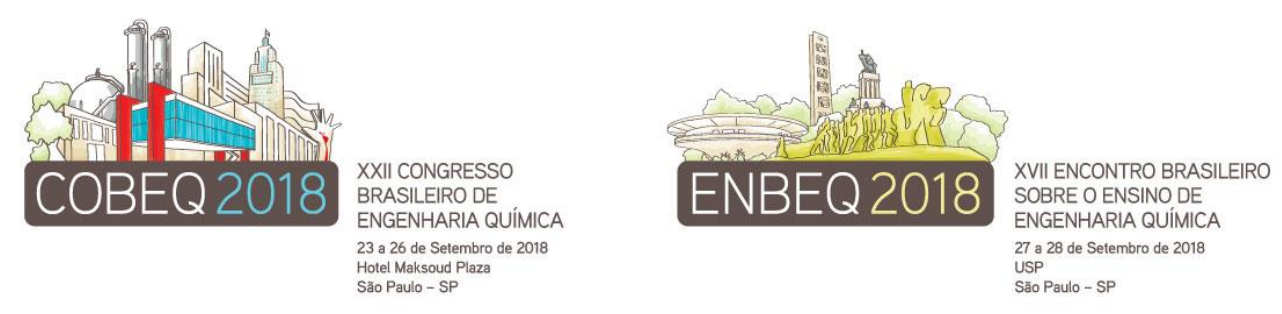

As parafinas, com entalpia de mudança de fase entre 100 a $250 \mathrm{~J} / \mathrm{g}$, possuem vantagens como PCMs com: baixa toxicidade; compatíveis com recipientes metálicos; quimicamente estáveis; baixo sub-resfriamento e suas misturas podem ser baratas (STAMATIOU et al., 2017). Apesar de muito estudada, a taxa de transporte de calor através das parafinas durante a cristalização não é bem conhecida, porque depende da morfologia da camada em formação. Nesta pesquisa este aspecto foi estudado teoricamente para uma geometria de "dedo frio", uma configuração adequada para estudos experimentais sobre a morfologia da camada.

\section{METODOLOGIA}

Foi elaborado um modelo matemático para prever a transferência de calor durante a cristalização de um PCM ao redor de um "dedo frio" ou "cold finger" cilíndrico, o qual é constituído de dois tubos de cobre concêntricos, sendo uma das extremidades do tubo externo fechada. No dedo frio, internamente, circula um fluido frio que mantém a superfície externa com a temperatura baixa (abaixo da temperatura de fusão) e controlada por banho termostático. Ele é submerso na substância fundida que se intenciona estudar, sendo que na modelagem o volume desse fluido foi considerado infinito. A substância fundida é ajustada na temperatura desejada (de fusão ou superior) através da circulação de um fluido de aquecimento por uma camisa externa ao recipiente, também controlada por um banho termostático. A temperatura do fluido quente representa a da adsorção do gás no tanque e a do fluido frio ao do local que o cilindro está.

\subsection{Equacionamento}

A taxa de condução de calor pelo PCM sólido será:

$\dot{Q}=k_{P C M} \cdot 2 . \pi \cdot L \cdot \frac{T_{r s l}-T_{r e}}{\ln \left(\frac{r_{e}}{r_{s l}}\right)}$

Analogamente, aplicando-se a equação de conservação de energia para a parede do dedo frio:

$\dot{Q}=k_{d} \cdot 2 \cdot \pi \cdot L \cdot \frac{T_{r e}-T_{r i}}{\ln \left(\frac{r_{i}}{r_{e}}\right)}$

$\mathrm{Na}$ interface sólido-líquido, onde $\mathrm{T}=\mathrm{T}_{\mathrm{m}}$, a massa de PCM solidificado no dado intervalo de tempo é proporcional ao calor latente de fusão do PCM.

$\dot{Q}=\frac{m_{s}}{\Delta t} \cdot \Delta H_{s l}$

A massa de PCM solidificada no intervalo de tempo deposita-se na superfície externa do dedo frio, aumentando a espessura de sólido de acordo com a seguinte expressão:

$r_{\text {sl }}$ final $=\sqrt{\left(r_{\text {slinicial }}^{2}+\frac{m_{s}}{\rho_{s} \cdot \pi \cdot L}\right)}$

Ainda, na parede interna do dedo frio, por onde circula o fluido refrigerante, pode-se escrever:

$\dot{Q}=h_{c} \cdot 2 \cdot \pi \cdot r_{i} \cdot L \cdot\left(T_{i}-T_{c}\right)$ 

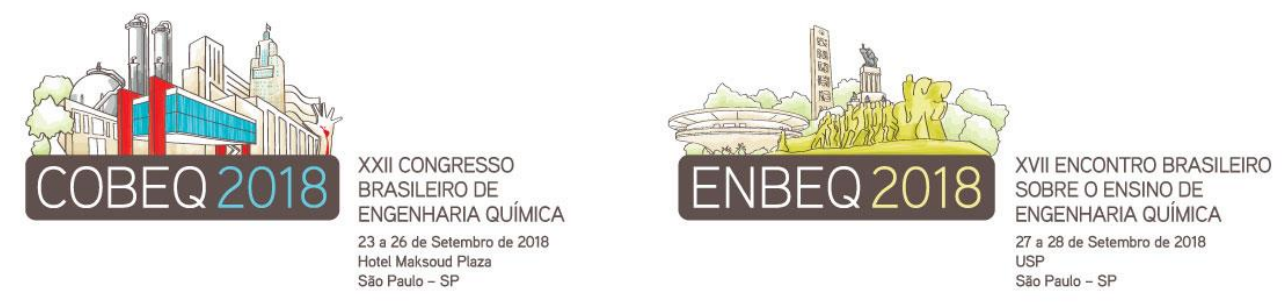

\section{RESULTADO E DISCUSSÃO}

Simulações foram feitas para mistura parafínica com propriedades obtidas em literatura: condutividade térmica do cobre $401 \mathrm{~W} / \mathrm{m} . \mathrm{K}$; fluido de resfriamento sendo água a $25{ }^{\circ} \mathrm{C}$ (MALONEY, 2008); condutividade térmica da parafina 0,240 W/m.K; calor específico da parafina $2890 \mathrm{~J} / \mathrm{kg} . \mathrm{K}$; densidade da parafina sólida $900 \mathrm{~kg} / \mathrm{m}^{3}$ (INCROPERA et al. 2007); calor latente de cristalização da parafina 170 kJ/kg (AGARWAL; SARVIYA, 2017).

A temperatura do fluido quente foi fixada como igual à temperatura de fusão de $50{ }^{\circ} \mathrm{C} \mathrm{e}$ a temperatura do fluido frio foi $25{ }^{\circ} \mathrm{C}$. A figura 1A mostra que a massa depositada no dedo frio aumenta mais rapidamente no início da cristalização. Isso se deve à maior taxa de transferência de calor inicial, mostrada na figura 1B, por dois efeitos: a baixa resistência térmica do cobre e o diminuto volume de parafina cristalizada, que aumenta a resistência térmica (figura 1C) à medida que em que é formada. A posição da interface, presente na figura 1D, confirma o comportamento das figuras 1A e 1B. Como o meio fundido é infinito, a massa cristalizada continua a aumentar indefinidamente a taxas de transferência de calor sucessivamente menores. Em certas condições de cristalização, a morfologia da camada sólida pode resultar em condutividade térmica reduzida, o que provoca um aumento no tempo de cristalização. Por exemplo, o tempo para formação de uma camada de $15 \mathrm{~mm}$ de parafina foi aumentado de 33 para 48 min com a redução à metade da condutividade térmica.
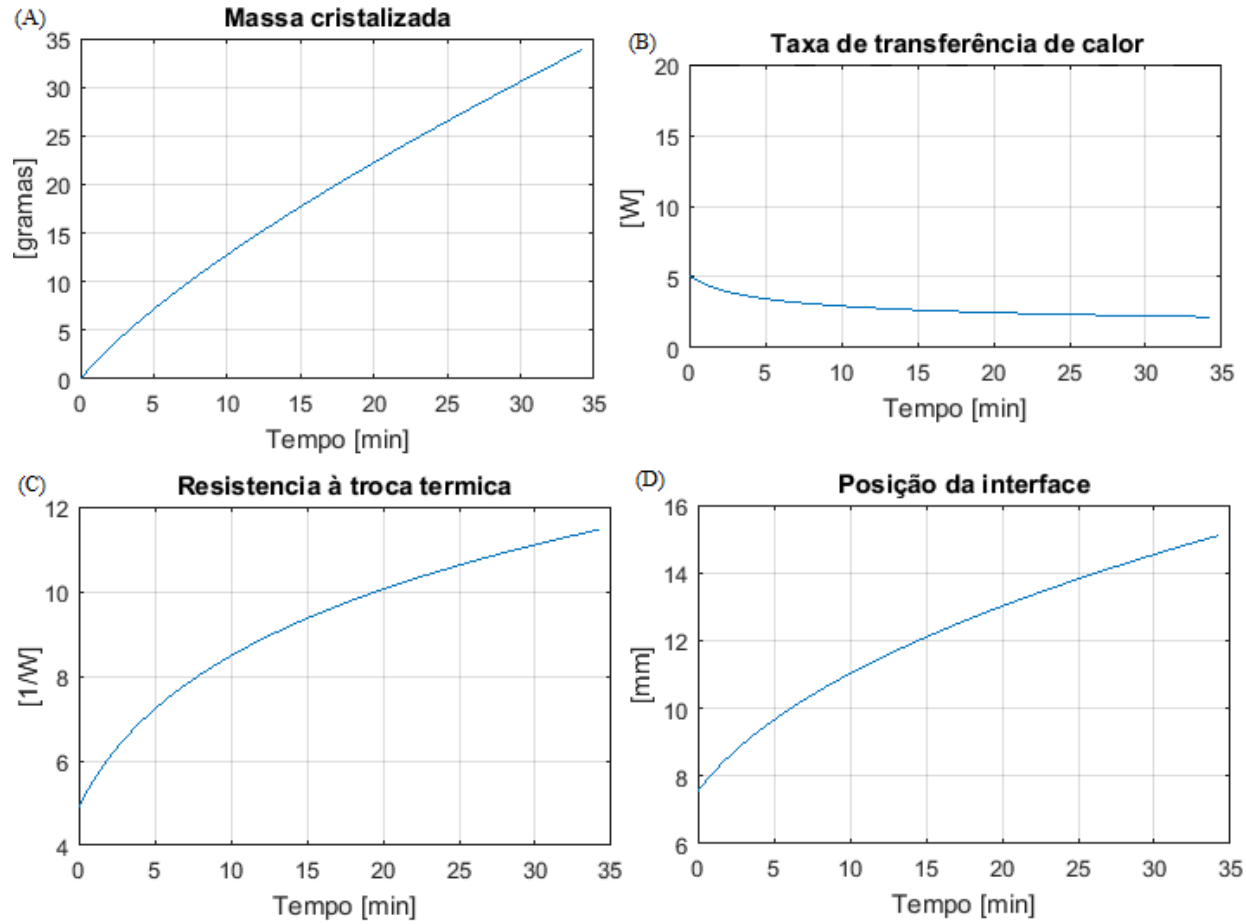

Figura 1 -(A) Curva da massa cristalizada ao redor do dedo frio; (B) Taxa de transferência de calor do sistema até a interface sólida. (C) Curva da resistência à troca térmica do sistema até a interface sólida. (D)

\section{CONCLUSÕES}

A taxa de transferência de calor de um PCM parafínico foi estudada teoricamente em 


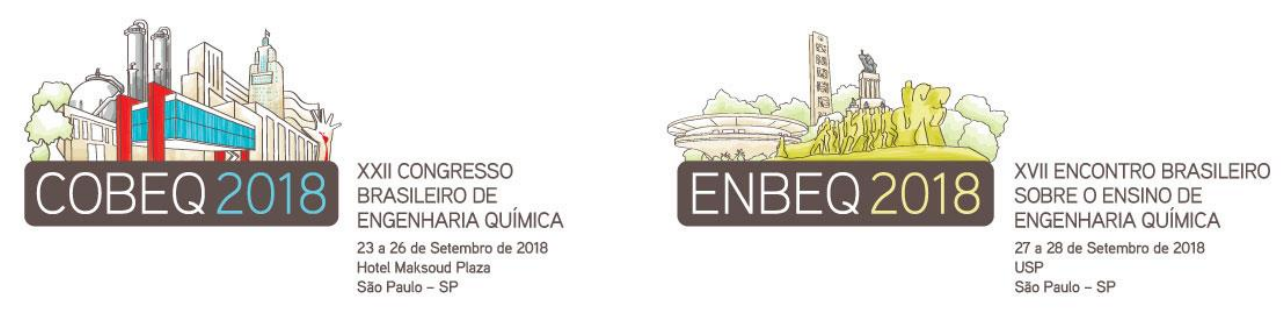

uma configuração de "dedo frio". Foi mostrado como a condutividade da camada sólida afeta a taxa de transferência de calor do PCM. Caso a forma cristalina modifique-se, a mudança na condutividade influenciará no tempo de cristalização do PCM e consequentemente na velocidade de absorção ou liberação de energia durante a mudança de fase.

\section{NOMENCLATURA}

\begin{tabular}{l|l}
\hline $\mathrm{k}_{\mathrm{PCM}}-$ condutividade térmica do PCM, W/m.K. & $\mathrm{L}$ - comprimento do dedo frio, $\mathrm{m}$. \\
$\mathrm{k}_{\mathrm{d}}$ - condutividade térmica do dedo frio, W/m.K. & $\mathrm{h}_{\mathrm{c}}-$ coef. convectivo, $\mathrm{W} / \mathrm{m}^{2} . \mathrm{K}$ \\
$\dot{\mathrm{Q}}$ - taxa de transferência de calor. W. & $\mathrm{m}_{\mathrm{s}}-$ massa sólida, $\mathrm{kg}$. \\
$\mathrm{r}_{\mathrm{sl}}$ - espessura da camada de PCM sólido, $\mathrm{m}$. & $\rho_{\mathrm{s}}-$ densidade do $\mathrm{PCM}, \mathrm{kg} / \mathrm{m}^{3}$. \\
$\mathrm{r}_{\mathrm{i}}$ - raio interno do dedo frio, $\mathrm{m}$. & $\mathrm{T}_{\mathrm{i}}-$ temperatura da saída dedo frio, $\mathrm{K}$. \\
$\mathrm{r}_{\mathrm{e}}-$ raio externo do dedo frio, m. & $\mathrm{T}_{\mathrm{c}}-$ temperatura da entrada dedo frio, $\mathrm{K}$ \\
$\Delta \mathrm{H}_{\mathrm{sl}}-$ calor latente de cristalização $\mathrm{J} / \mathrm{kg}$. & $\mathrm{T}_{\mathrm{re}}-$ temperatura no $\mathrm{r}_{\mathrm{e}}, \mathrm{K}$ \\
$\Delta \mathrm{t}-$ tempo decorrido, s. & $\mathrm{T}_{\mathrm{ri}}-$ temperatura no $\mathrm{r}_{\mathrm{i}} \mathrm{K}$ \\
\hline
\end{tabular}

\section{REFERÊNCIAS}

AGARWAL, A.; SARVIYA, R. M. Characterization of Commercial Grade Paraffin wax as Latent Heat Storage material for Solar dryers. Materials Today: Proceedings, v. 4, n. 2, p. 779-789, 2017. Disponível em: 〈http://dx.doi.org/10.1016/j.matpr.2017.01.086>.

EL-SHARKAWY, I. I.; MANSOUR, M. H.; AWAD, M. M.; EL-ASHRY, R. Investigation of Natural Gas Storage through Activated Carbon. Journal of Chemical \& Engineering Data, v. 60, n. 11, p. 3215-3223, 2015. Disponível em:

<http://pubs.acs.org/doi/10.1021/acs.jced.5b00430>.

LI, X.; LI, Y. Applications of organic phase change materials embedded in adsorbents for controlling heat produced by charging and discharging natural gas. Adsorption, v. 21, n. 5, p. 383-389, 2015.

MALONEY, J. O. Perry's Chemical Engineers' Handbook. [s.1.] McGraw-Hill Education, 2008.

STAMATIOU, A.; OBERMEYER, M.; FISCHER, L. J.; SCHUETZ, P.; WORLITSCHEK, J. Investigation of unbranched, saturated, carboxylic esters as phase change materials.

Renewable Energy, v. 108, p. 401-409, 1 ago. 2017. Disponível em: <https://www.sciencedirect.com/science/article/pii/S0960148117301428>. Acesso em: 20 fev. 2018.

ZAKARIA, Z.; GEORGE, T. the Performance of Commercial Activated Carbon Absorbent for Adsorbed Natural Gas Storage. International Journal of Research and Reviews in Applied Sciences, v. 9, n. 2, p. 225-230, 2011. 